D I A $\quad$ L

Développement et insertion internationale

DOCUMENT DE TRAVAIL

DT/2001/15

\title{
Taxation optimale de la consommation et biens informels
}

Jean-François GAUTIER 


\title{
TAXATION OPTIMALE DE LA CONSOMMATION ET BIENS INFORMELS ${ }^{1}$
}

\author{
Jean-François Gautier \\ (Université Paris IX et DIAL) \\ e-mail : gautier1@caramail.com
}

\section{Document de travail DIAL / Unité de Recherche CIPRE \\ Octobre 2001}

\section{RESUME}

La question posée par ce papier est la suivante : dans quelle mesure est-il souhaitable de taxer le secteur informel? Un modèle théorique de taxation optimale permet d'abord de fournir une réponse analytique à cette question. Une version empirique du modèle permet ensuite d'estimer les taux de taxation optimaux à Madagascar. On montre ainsi que la taxation optimale des biens informels est toujours très inférieure à celle des biens formels. Néanmoins, pour des degrés de progressivité «raisonnables », le taux de taxation optimal moyen du secteur informel est nettement plus important que le taux effectif actuel. Toutefois, la solution optimale peut être de subventionner le secteur informel pour des degrés élevés de progressivité.

\section{ABSTRACT}

This paper analyses whether it is worth taxing the black market. A theoretical optimal tax model provides a first analytical discussion. An empirical version allows us to estimate an optimal tax system for Madagascar. For reasonable inequality aversion parameters, the taxation's rate of the informal good is always quite below the formal good's one. Nevertheless, tax rates on informal goods are above the actual rates. Informal goods could be optimally subsidised for higher inequality aversion parameters.

Classification JEL : H21 (Efficiency, Optimal Taxation) ; H26 (Tax Evasion).

\footnotetext{
${ }^{1}$ Je remercie Rachel Ravelosoa, François Roubaud et Charlotte Guénard d'avoir bien voulu me communiquer les données et documents techniques de l'enquête budget - consommation des ménages à Antananarivo (MADIO 1995b), ainsi que l'équipe DIAL pour les commentaires apportés à une version précédente de ce papier.
} 


\section{Table des matières}

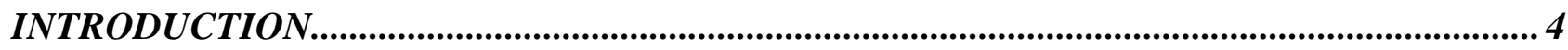

1. FISCALITE DES BIENS ET CONSOMMATION DES MENAGES .............................5

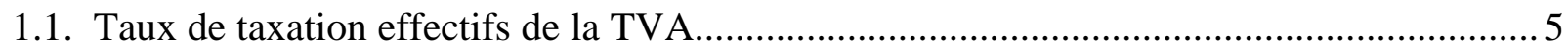

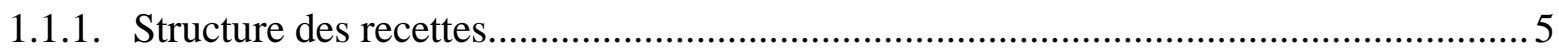

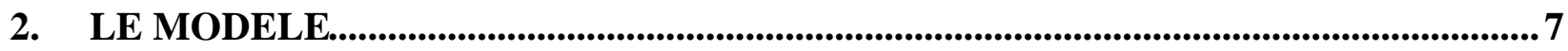

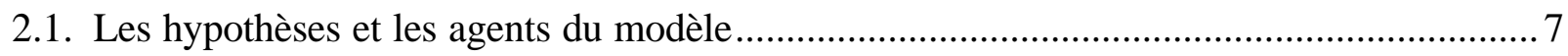

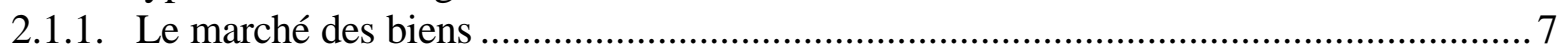

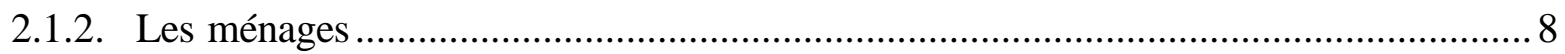

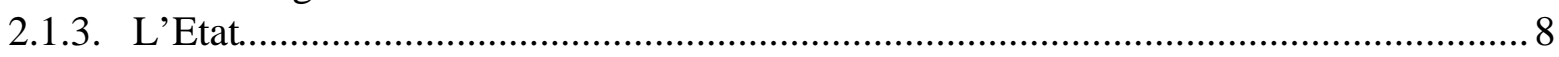

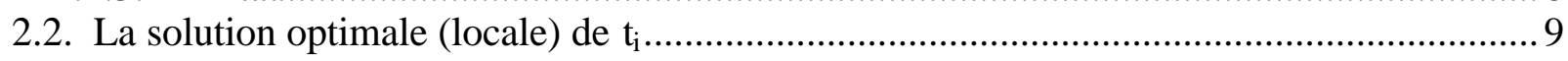

2.2.1. Relation entre $t_{i}$ et la dérivée de la demande compensée aux prix $\left(S_{b_{i}, b_{i}}^{h}\right) \ldots \ldots \ldots \ldots \ldots \ldots . \ldots 11$

2.2.2. Relation entre ti et la dérivée de la demande au revenu.......................................... 11

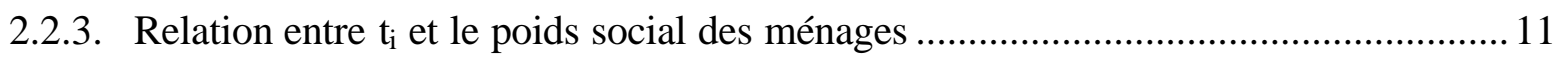

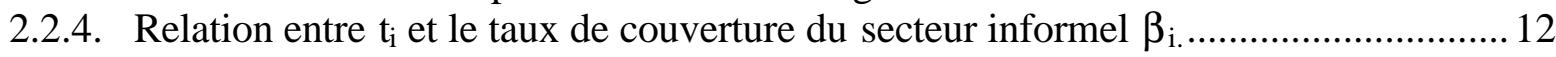

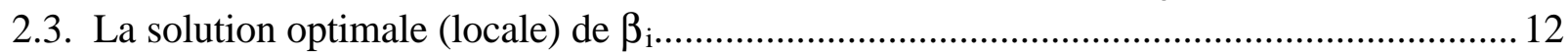

2.3.1. Commentaires, cas d'une taxation optimale du secteur informel............................. 13

2.3.2. Cas où il est optimal de subventionner le secteur informel.................................... 13

3. MODELE EMPIRIQUE ET SIMULATIONS................................................................... 14

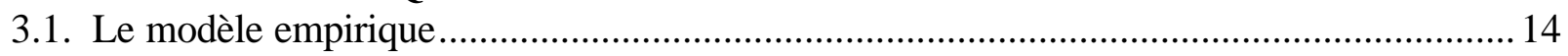

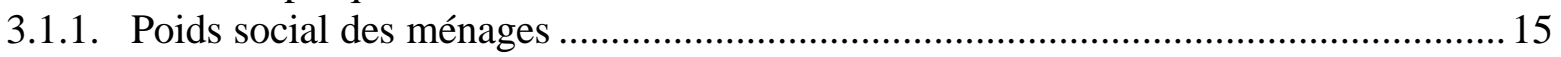

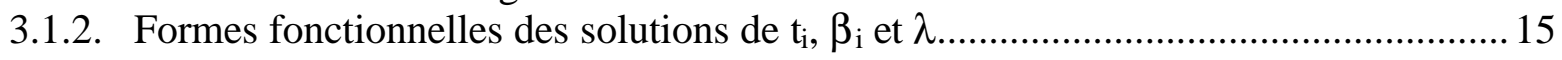

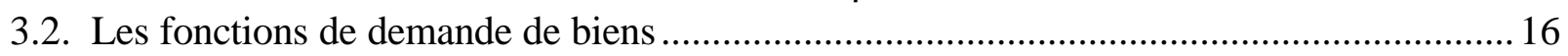

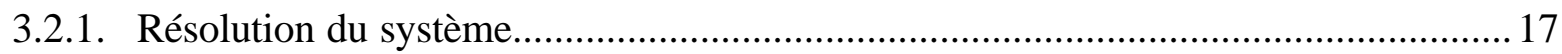

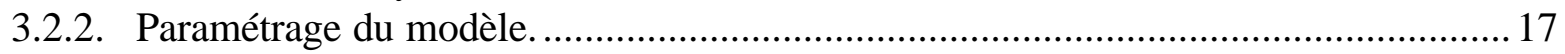

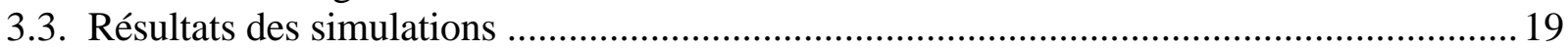

3.3.1. Taxation des biens formels versus taxation des biens informels. ............................. 20

3.3.2. Résultats sur la taxation des biens et services............................................... 21

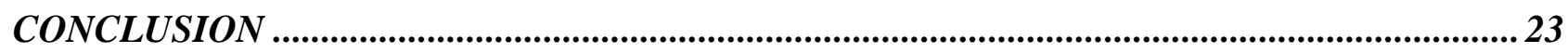

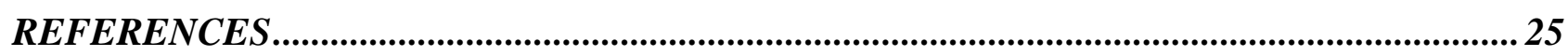

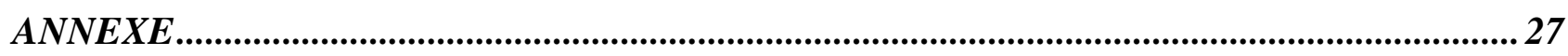

\section{Liste des tableaux}

Tableau $n^{\circ} 1-1$ : Taux de pression estimé de la TVA par produits.................................................. 6

Tableau $n^{\circ}$ 1-2 : Structure de la consommation par quintiles de dépenses ${ }^{*}$...................................... 6

Tableau $n^{\circ}$ 3-1 : Elasticités prix et revenus et distribution de la demande ...................................... 18

Tableau $n^{\circ}$ 3-2 : Taux de taxation optimaux avec demande endogène ............................................. 23

\section{Liste des Annexes}

Annexe $n^{\circ} 1:$ Variation de la demande et des prix........................................................................... 27

Annexe $n^{\circ} 2$ : Structure de la consommation et taux de pression par déciles de dépenses............... 27 


\section{INTRODUCTION}

L'objet de ce papier est d'estimer une structure optimale de taux de taxation sur les biens de consommation et les services. L'originalité est de prendre en compte la dualité sur le marché des biens et services, en distinguant les biens formels des biens informels. L'intérêt d'intégrer cette dualité réside dans le fait que les marchés des biens et services dans les PED sont divisés entre un marché formel, assujetti aux impôts, et un marché informel faiblement taxé. Par ailleurs, la demande relative pour chaque type de bien (formel / informel) varie avec les revenus des consommateurs.

Cette dualité entraîne ainsi une distorsion sur les marchés qui théoriquement doit être à l'origine d'une perte d'efficacité de l'économie. Elle entraîne en effet une réallocation «artificielle »des facteurs de production vers le secteur informel et motive de plus les comportements de fraude fiscale. Ainsi, taxer les biens informels permettrait par exemple, à revenu de l'Etat constant, de diminuer les taux de taxation nominaux sur le secteur formel. Cette baisse des taux réduirait la taille $\mathrm{du}$ «poids mort » engendré par la fiscalité sur les marchés formels et par conséquent améliorerait l'efficacité du système de taxation.

Si du point de vue de l'efficacité des marchés, l'Etat doit rechercher la taxation du secteur informel, le principe de redistribution devrait en revanche l'en écarter. En effet, le bien informel semble être un bien nécessaire, voire inférieur dans les PED, i.e. dont la part dans la consommation décroît avec le revenu des ménages [MADIO (1995b), Roubaud (1994), Cogneau et al. (1996), Boyabé (1999)]. La taxation du bien informel accroîtrait donc principalement la taxation du budget des ménages les plus démunis. D'autre part, les modèles de fiscalité optimale avec fraude [voir notamment Kaplow (1990) et Cremer et Gahvary (1993), voir Ray (1997) pour une revue] montrent que la taxation des marchés avec fraude doit être inférieure à celle des marchés sans fraude. L'existence d'un coût lié à la fraude, croissant avec la taxation, explique ce résultat qui permet alors de réduire le poids mort sur le marché frauduleux (critère d'efficacité). Toutefois ces modèles ne prennent pas en compte la substituabilité des biens entre les marchés formels et informels. La perte d'efficacité sur les marchés formels est alors sous-estimée. En outre, leur cadre d'agent représentatif ne leur permet pas d'étudier les effets redistributifs de la taxation de la consommation.

En tenant compte des deux critères d'efficacité et d'équité, nous allons chercher dans ce papier à définir une fiscalité optimale qui permettra d'estimer dans quelle mesure l'Etat doit fiscaliser le secteur informel par rapport au secteur formel. Pour traiter ces questions, nous avons choisi de suivre le plan suivant :

Une première partie présente les taux de taxation des biens formels et informels, et la structure de la consommation par revenu, qui servent à calibrer notre modèle. Les statistiques utilisées sont : l'enquête consommation des ménages à Antananarivo et l'enquête sur le secteur informel (MADIO 1995). Nous traitons aussi les données de l'administration fiscale sur les recettes de TVA prélevées dans l'agglomération d'Antananarivo en 1998.

Nous construisons ensuite un modèle théorique de taxation optimale avec un vecteur de biens formels et un autre de biens informels. Nous développons analytiquement ce modèle de manière à présenter comment les taux de taxation des secteurs formels et informels sont influencés par la typologie des biens.

Une version empirique de ce modèle est ensuite présentée. Elle permet d'estimer un système de taxation optimale de la consommation sur la base d'enquête sur les ménages à Madagascar (MADIO 1995b) et de valeurs d'élasticités prix et revenus tirées de la littérature. 


\section{FISCALITE DES BIENS ET CONSOMMATION DES MENAGES}

Cette première partie a pour but de présenter le système actuel de TVA à Madagascar. On cherche essentiellement à estimer comment les exonérations et le secteur informel jouent sur le taux de pression effectif des biens (par rapport aux taux prévus par la loi). En utilisant les enquêtes sur la consommation des ménages, on produit alors une première information sur le caractère redistributif de la TVA.

\subsection{Taux de taxation effectifs de la TVA²}

\subsubsection{Structure des recettes}

La TVA est actuellement la première source de revenus fiscaux à Madagascar, avec $31 \%$ du total des recettes fiscales de l'administration centrale. Toutefois ces recettes représentaient en 1998 un peu moins de $3 \%$ du PIB. Elle est appliquée à un taux unique de $20 \%$ (depuis la loi de finances de 1996), accompagnée d'exonérations permettant de ne pas augmenter le prix des produits «sensibles ». Le Code Général des Impôts (CGI) prévoit principalement l'exonération des produits alimentaires non transformés, les secteurs de l'éducation et de la santé ${ }^{3}$. Il semble de plus que des exonérations puissent être octroyées à des entreprises de manière discrétionnaire. In fine, on estime à partir des fichiers de l'administration fiscale que $40 \%$ du chiffre d'affaires des entreprises formelles est actuellement exonéré.

Bien que le système de TVA prévu par la loi soit relativement simple, l'accumulation des exonérations et autres régimes dérogatoires sont à l'origine de taux de taxation multiples (cf. tableau 1-1). Les exonérations modifient le schéma de la TVA de la manière suivante :

- D'une part, elles ont pour effet de réduire le taux de taxation des biens normalement taxés au taux de $20 \%$ (lorsqu'elles sont accordées de manière nominative à des entreprises). Par exemple, le taux de pression moyen sur l'habillement qui devrait normalement être de 16,7\% (avec exonérations sur produits uniquement), ne serait en réalité que de 3,7\% sur les biens formels.

- D'autre part, les secteurs exonérés supportent une charge rémanente de TVA dès qu'ils se fournissent auprès d'entreprises assujetties (ces entreprises n'étant pas autorisées à récupérer la TVA payée, cf. Gautier 1999). Par exemple, le tableau 1-1 montre que les céréaliers, l'éducation, la santé qui sont normalement des secteurs exonérés, supportent toutefois une TVA.

In fine la somme de ces mécanismes réduit le taux de pression de la TVA sur les biens et services formels. Le taux effectif moyen des produits formels, qui est actuellement de $8,0 \%$, serait de $11,3 \%$ si le code général des impôts était parfaitement appliqué (sans rémanences et sans exonérations nominales).

En outre, le taux de taxation rémanent des biens informels serait de 0,3\%. In fine, le taux de pression moyen, qui devrait être de 7,5\% lorsqu'il est estimé sur la base des termes du CGI, est en réalité de 3,2\% dans l'agglomération d'Antananarivo ${ }^{4}$.

\footnotetext{
${ }^{2}$ La méthode d'estimation des taux effectifs de TVA et celle d'estimation de la consommation des biens informels par les ménages sont présentées en annexe de ce papier.

${ }^{3}$ Cf. encadré en annexe pour plus de détail.

${ }^{4}$ Ce résultat semble cohérent avec le taux de pression calculé à partir du TOFE et du PIB qui est de 2,8\% sur l'ensemble du territoire.
} 
Tableau $n^{\circ}$ 1-1 : Taux de pression ${ }^{5}$ estimée de la TVA par produits

\begin{tabular}{|l|c|c|c|c|}
\hline \multirow{2}{*}{ Biens } & \multicolumn{3}{|c|}{ Taux effectif } & Taux sur la base \\
\cline { 2 - 4 } du code des impôts
\end{tabular}

Sources: Centre Fiscal des Entreprises, MADIO, calculs propres, estimés à partir des données fiscales de 1998.

Le tableau 1-2 permet de présenter l'évolution de la structure de consommation des ménages en fonction de leur revenu. On observe que :

- La part budgétaire des biens et services informels est une fonction décroissante des dépenses des ménages. Si elle représente un peu moins de $76 \%$ de la consommation du premier quintile de dépense, cette part n'est plus que de $55 \%$ pour le dernier quintile. Les biens informels seraient donc des biens nécessaires, voir inférieures. De fait, la consommation de biens informels doit favoriser la progressivité du système de taxation, ces produits étant moins taxés que les biens formels.

- Les préférences des consommateurs évoluent avec leur niveau total de dépenses. Si les biens alimentaires sont des biens nécessaires ; l'habillement, le transport, la santé, l'éducation et les autres biens et services sont visiblement des biens de luxe. La part du logement et des équipements reste stable dans la consommation. Toutefois, l'apparente stabilité du logement peut être biaisée par le fait que les loyers n'ont pas été imputés au budget des ménages propriétaires de leur logement. Ne pas imputer les loyers est justifié dans notre cas puisque nous nous intéressons uniquement aux dépenses monétaires effectivement ou potentiellement taxées.

\section{Tableau $\mathbf{n}^{\circ}$ 1-2 : Structure de la consommation par quintiles de dépenses*}

\begin{tabular}{|l|c|c|c|c|c|}
\hline Quintiles de dépenses & 1 & 2 & 3 & 4 & 5 \\
\hline Riz, céréales & $31.1 \%$ & $28.5 \%$ & $24.3 \%$ & $20.5 \%$ & $8.1 \%$ \\
Fruits, légumes, élevage & $18.9 \%$ & $20.4 \%$ & $21.9 \%$ & $21.8 \%$ & $16.9 \%$ \\
Alimentation transformée & $15.9 \%$ & $13.5 \%$ & $14.2 \%$ & $14.6 \%$ & $10.7 \%$ \\
Habillement & $5.6 \%$ & $5.8 \%$ & $5.7 \%$ & $6.6 \%$ & $7.9 \%$ \\
Logement & $13.3 \%$ & $14.1 \%$ & $14.5 \%$ & $13.8 \%$ & $12.2 \%$ \\
Equipement, entretien maison & $5.2 \%$ & $5.9 \%$ & $5.6 \%$ & $5.7 \%$ & $5.3 \%$ \\
Santé & $3.2 \%$ & $3.4 \%$ & $5.1 \%$ & $3.9 \%$ & $5.4 \%$ \\
Transport & $4.1 \%$ & $4.6 \%$ & $4.2 \%$ & $6.0 \%$ & $24.7 \%$ \\
Education & $1.7 \%$ & $2.3 \%$ & $3.3 \%$ & $4.9 \%$ & $3.7 \%$ \\
Autres biens \& services & $1.0 \%$ & $1.6 \%$ & $1.4 \%$ & $2.2 \%$ & $5.2 \%$ \\
Part des biens informels & $75.8 \%$ & $72.3 \%$ & $67.7 \%$ & $63.7 \%$ & $55.0 \%$ \\
Taux de taxation moyen & $2.0 \%$ & $2.3 \%$ & $2.3 \%$ & $2.6 \%$ & $3.8 \%$ \\
\hline
\end{tabular}

Sources: Centre Fiscal des Entreprises, MADIO, calculs propres, estimés à partir des données fiscales de 1998.

* ni les loyers des ménages propriétaires de leur logement, ni l'autoconsommation n’ont été imputés dans les dépenses des ménages.

\footnotetext{
${ }^{5}$ Le taux de pression est calculé sur le prix au consommateur : Tx conso $=$ Tx producteur $/(1+$ Tx producteur). Pour un taux de $20 \%$ sur le prix producteur équivaut à un taux d'environ $16,7 \%$ sur le prix consommateur.
} 
Ainsi, l'évolution des préférences des consommateurs en fonction des revenus entraîne bien une redistribution verticale de la taxation des biens. Le taux moyen de taxation de la consommation des ménages les plus pauvres étant de $2,0 \%$, lorsqu'il est de $3.8 \%$ pour le quintile le plus riche.

\section{LE MODELE}

\subsection{Les hypothèses et les agents du modèle}

Notre modèle s'inscrit dans la tradition des modèles de fiscalité indirecte optimale dans un cadre multi-agents (Diamond et Mirrlees 1971). Nous nous inspirons de Kaplow (1990) et de Cremer et Ghavary (1993) pour intégrer une dualité dans notre modèle entre d'une part des biens formels parfaitement taxés et d'autre part des biens informels imparfaitement taxés. Seule l'introduction de cette dualité entre les biens de consommation apporte une modification aux hypothèses standards de la littérature de la fiscalité optimale.

\subsubsection{Le marché des biens}

Nous supposons que le marché des biens est caractérisé par «n»paires de biens «i $»$. Les paires de biens traduisent la dualité entre les secteurs formels et informels au sein de l'économie. Autrement dit, pour chaque bien « $\mathrm{i}$ », il existe un bien $\left(\mathrm{x}_{\mathrm{i}}\right)$ produit par le secteur formel et un autre bien $\left(\mathrm{y}_{\mathrm{i}}\right)$ produit par le secteur informel. Au total, il existe 2.n biens dans l'économie.

Nous supposons que les biens formels sont taxés au taux $t_{i}$. Les biens informels le sont au taux de : $\beta_{\mathrm{i}} \mathrm{t}_{\mathrm{i}}$. La variable $\beta_{\mathrm{i}}$ représente le taux de fiscalisation (ou de couverture) du secteur informel dans la branche i. Autrement dit, $\left(1-\beta_{i}\right)$ est le taux de fraude fiscale du secteur informel. Ainsi, si $\beta_{i}=0$, le secteur informel dans la branche i échappe intégralement à la taxation de sa production. L'Etat n'a pas la capacité de taxer le chiffre d'affaires des micro-unités de production. En revanche, si $\beta$ i tend vers 1, l'Etat s'est donné les moyens de limiter la fraude fiscale des micro-entreprises. La production $\mathrm{du}$ «secteur informel » est taxée à un taux proche du taux normal prévu par le code des impôts.

Ainsi, sur le marché des biens, les prix au consommateur peuvent s'écrire :

Pour les biens $\mathrm{x}_{\mathrm{i}}: \quad \mathrm{q}_{\mathrm{xi}}=\mathrm{p}_{\mathrm{xi}}\left(1+\mathrm{t}_{\mathrm{i}}\right)$

Pour les biens $y_{i}: \quad q_{y i}=p_{y i}\left(1+\beta_{i} t_{i}\right)$

Où $\mathrm{p}_{\mathrm{xi}}$ et $\mathrm{p}_{\mathrm{yi}}$ sont les prix au producteur. Sous l'hypothèse de concurrence pure et parfaite, les prix au producteur sont égaux au coût marginal et les consommateurs sont «price-taker». Par simplification, on suppose donc que les prix au producteur sont exogènes. Autrement dit, nous ne tenons pas compte de l'effet produit par la taxation des intrants sur le coût des entreprises (rémanence de la TVA sur le secteur informel et les entreprises exonérées). Pour simplifier notre exposé, nous posons par la suite que les prix au producteur sont normés à 1 .

Pour la clarté de l'exposé, les notations utilisées sont les suivantes : $\mathrm{X}$ est le vecteur colonne des $\mathrm{x}_{\mathrm{i}}$, et Y le vecteur colonne des $y_{i}$. B est la matrice $(n, 2)$ définie par B: $(X, Y)$. Ainsi, on notera $b_{i}$ la matrice ligne définie par la paire de biens $\left(x_{i}, y_{i}\right)$. Par ailleurs, pour l'ensemble des $n$ branches de l'économie, on notera $\beta$ le vecteur colonne formé par l'ensemble des $\beta_{\mathrm{i}}$. 


\subsubsection{Les ménages}

L'utilité directe des ménages est une fonction de la consommation de ces paires de biens. Pour simplifier, on suppose que le travail (loisir) et les biens de consommation sont séparables dans la fonction d'utilité du ménage. De même, nous admettrons que les ménages ne font que consommer, i.e. ils n'ont pas d'activités productives ou commerçantes. Ces deux dernières hypothèses permettent de supposer une contrainte budgétaire linéaire par rapport au taux de taxation, facilitant ensuite le traitement analytique du modèle.

En outre, on suppose que les biens formels et informels sont imparfaitement substituables dans la demande des ménages. Cette hypothèse se justifie par la différence observée de qualité et de mode de commercialisation des deux biens.

On pose $\mathrm{U}^{\mathrm{h}}$ l'utilité (Marshalienne) du consommateur h, avec $\mathrm{h}:(1, \ldots, \mathrm{H})$.

$$
\mathrm{U}^{\mathrm{h}}=\mathrm{U}^{\mathrm{h}}\left(\mathrm{X}^{\mathrm{h}}, \mathrm{Y}^{\mathrm{h}}, \mathrm{T}^{\mathrm{h}}\right)
$$

Avec, pour le ménage $h, \mathrm{X}^{\mathrm{h}}$ le vecteur des consommations en biens $\mathrm{x}_{\mathrm{i}}, \mathrm{Y}^{\mathrm{h}}$ vecteur des $\mathrm{y}_{\mathrm{i}}$, et $\mathrm{T}^{\mathrm{h}}$ le temps de travail.

L'utilité indirecte (Hicksienne) s'écrit :

$\mathrm{V}^{\mathrm{h}}=\mathrm{V}^{\mathrm{h}}\left(\mathrm{q}_{\mathrm{x}}, \mathrm{q}_{\mathrm{y}}, \mathrm{I}^{\mathrm{h}}, \mathrm{w}^{\mathrm{h}}\right)$

Avec $\mathrm{I}^{\mathrm{h}}$ le revenu et $\mathrm{w}^{\mathrm{h}}$ le taux de salaire du ménage $\mathrm{h}$, qui sont tous deux supposés exogènes.

\subsubsection{L'Etat}

Si $\beta$ est le taux de fiscalisation des unités de production informelles, son niveau dépend des moyens mis en œuvre par les autorités pour fiscaliser les micro-entreprises informelles ${ }^{6}$. De fait, le coût de la collecte des impôts par l'administration fiscale est une fonction de la variable $\beta$. On nomme $g(\beta)$ la fonction de coût liée au taux de contrôle. Cette fonction est positive et croissante par rapport à $\beta$. On pourra aussi faire l'hypothèse qu'elle est convexe. Comme les recettes fiscales sont proportionnelles à $\beta_{\mathrm{i}}$, la convexité de la fonction de coûts permet ainsi de poser une productivité marginale décroissante des contrôles effectués par l'administration fiscale (i.e. l'efficacité des contrôles est décroissante). On a donc pour résumer : $g(\beta)>0$, $g^{\prime}(\beta)>0$ et $g^{\prime \prime}(\beta)>0$.

En théorie, l'objectif de l'Etat est donc de définir les taux de taxation $t_{i}$ et les taux de couverture des unités informelles $\beta_{i}$ de sorte à maximiser la fonction d'utilité sociale tout en atteignant son objectif de recettes fiscales. Le programme de l'Etat s'écrit alors :

La fonction d'utilité sociale s'écrit :

$$
\mathrm{W}=\mathrm{W}\left(\mathrm{V}^{1}, \ldots \ldots, \mathrm{V}^{\mathrm{H}}\right)
$$

W est la fonction d'utilité sociale. En supposant que celle-ci est de type «Bergson-Samuelson », les utilités indirectes de chaque ménage $\left(\mathrm{V}^{\mathrm{h}}\right)$ sont indépendantes les unes des autres (forme additive).

\footnotetext{
${ }^{6}$ On rappelle que cette fiscalisation n'intervient que par le biais du contrôle des unités de production informelle. Nous ne prenons pas en compte pour l'instant dans le modèle les phénomènes de rémanence qui permettent de taxer indirectement par les intrants la production informelle.
} 
Tandis que sa contrainte budgétaire est donnée par :

$$
R_{0}=\sum_{i=1}^{n} \sum_{h=1}^{H}\left(t_{i} x_{i}^{h}+\beta_{i} t_{i} y_{i}^{h}\right)-g(\beta)
$$

où $\mathrm{R}_{0}$ est l'objectif de recettes fiscales fixé par l'Etat. Le programme s'écrit :

$$
\left\{\begin{array}{l}
\max W=W\left(V^{1}, \ldots \ldots . ., V^{H}\right) \\
\text { s.c. } R o=\sum_{i=1}^{n} \sum_{h=1}^{H}\left(t_{i} x_{i}^{h}+\beta_{i} t_{i} y_{i}^{h}\right)-g(\beta)
\end{array}\right.
$$

Programme dont le Lagrangien s'écrit :

$$
L=W+\lambda\left[\sum_{i=1}^{n} \sum_{h=1}^{H}\left(t_{i} x_{i}^{h}+\beta_{i} t_{i} y_{i}^{h}\right)-g(\beta)-R o\right]
$$

On rappelle que les conditions du premier ordre se calculent par rapport à $t_{i}$ et $\beta_{i}$., i.e. les deux variables endogènes du système. Les expressions des solutions optimales de $t_{i}$ et $\beta_{i}$ uniquement en fonction des variables exogènes prennent une forme trop compliquée pour pouvoir être commentées de manière analytique. A l'instar des articles écrits antérieurement sur la fiscalité optimale avec fraude (cf. Kaplow 1990, Cremer et Ghavary 1993, Ray 1998), nous avons choisi de commenter les solutions en fonction de l'autre variable endogène. L'optimum étudié est donc local, c'est à dire exprimé en fonction de l'optimum de l'autre variable endogène ${ }^{7}$. Les applications numériques permettront par la suite de résoudre ce problème et de calculer des optima «absolus ».

\subsection{La solution optimale (locale) de $t_{i}$}

La condition du premier ordre par rapport à $t_{i} s^{\prime}$ écrit :

$$
\frac{\partial L}{\partial t_{i}}=0 \Leftrightarrow \sum_{h}\left(\frac{\partial W}{\partial V^{h}}\left[\frac{\partial V^{h}}{\partial q_{x_{i}}}+\frac{\partial V^{h}}{\partial q_{y_{i}}} \beta_{i}\right]\right)=-\lambda\left[\sum_{h}\left(x_{k}^{h}+\beta_{k} y_{k}^{h}\right)+\sum_{i} \sum_{h}\left(t_{i} \frac{\partial x_{i}^{h}}{\partial q_{b_{k}}}+\beta_{i}^{2} t_{i} \frac{\partial y_{i}^{h}}{\partial q_{b_{k}}}\right)\right]
$$

On notera que $\partial \mathrm{q}_{\mathrm{xi}}=\partial \mathrm{t}_{\mathrm{i}}$ et que $\partial \mathrm{q}_{\mathrm{yi}}=\beta_{\mathrm{i}} \partial \mathrm{t}_{\mathrm{i}}$, car les prix au producteur sont supposés exogènes dans le modèle.

Grâce à l'identité de Roy, nous savons que les propriétés de la fonction d'utilité indirecte permettent d'écrire :

$$
\frac{\partial V^{h}}{\partial q_{x_{i}}}=-\frac{\partial V_{k}^{h}}{\partial I^{h}} x_{k}^{h}=-\mu^{h} x_{k}^{h}
$$

$$
\frac{\partial V^{h}}{\partial q_{y_{i}}}=-\frac{\partial V_{k}^{h}}{\partial I^{h}} y_{k}^{h}=-\mu^{h} y_{k}^{h}
$$

Où $\mu^{\mathrm{h}}$ est l'utilité marginale du revenu du ménage h. Pour simplifier les écritures, nous posons :

$$
\Phi^{h}=\frac{\partial W}{\partial V^{h}} \mu^{h}
$$

\footnotetext{
${ }^{7}$ Cette méthode, quoique discutable, est celle appliquée dans la littérature : Kaplow (1990), Cremer et Ghavary (1993) procèdent de cette manière. Ray (1998) calcule la solution optimale qu'uniquement par rapport à $\mathrm{t}_{\mathrm{i}}$, considérant $\beta_{\mathrm{i}}$ fixé.
} 
$\Phi^{\mathrm{h}}$ représente l'effet d'une variation marginale de l'utilité revenu d'un ménage sur l'utilité sociale. Selon l'expression de Diamond et Mirrlees (1971), $\Phi^{\mathrm{h}}$ est «l'utilité sociale marginale du revenu du ménage $h »$.

De plus, l'équation de Slutsky permet d'écrire :

$$
\begin{gathered}
\frac{\partial x_{i}^{h}}{\partial q_{b_{k}}}=S_{b_{i}, b_{k}}^{h}-x_{k}^{h} \frac{\partial x_{i}^{h}}{\partial I^{h}} \\
\frac{\partial y_{i}^{h}}{\partial q_{b_{k}}}=S_{b_{i}, b_{k}}^{h}-y_{k}^{h} \frac{\partial y_{i}^{h}}{\partial I^{h}}
\end{gathered}
$$

Où $S_{b_{i}, b_{k}}^{h}$ est la matrice de Slutsky pour chaque ménage, c'est à dire la matrice des élasticités prix directes et croisées pour chaque bien de la demande compensée du ménage h. Etant donné que notre intérêt se porte essentiellement sur la relation entre les biens formels et informels, on peut restreindre notre cadre d'analyse en supposant que les biens ne sont substituables qu'à l'intérieur d'une même paire de biens $\left(\mathrm{x}_{\mathrm{i}}, \mathrm{y}_{\mathrm{i}}\right)$. La matrice de Slutsky se limite donc aux élasticités prix directs de chaque bien et aux élasticités prix croisés entre les biens informels et les biens formels d'une même catégorie de bien $\mathrm{i}$. Autrement dit, les élasticités prix croisés entre $\left(\mathrm{x}_{\mathrm{i}}, \mathrm{x}_{\mathrm{k}}\right)$ ou $\left(\mathrm{x}_{\mathrm{i}}, \mathrm{y}_{\mathrm{k}}\right)$ ou $\left(\mathrm{y}_{\mathrm{i}}\right.$, $\mathrm{y}_{\mathrm{k}}$ ) sont supposées être nulles. Cette hypothèse ne paraît pas être une restriction forte par rapport à la réalité. En effet, les études de Ravelosoa et al. (1999) à Madagascar, ou de Deaton et Grimard (1993) au Pakistan, montrent toutes deux, à partir de systèmes AIDS appliqués à la demande de biens alimentaires, que les élasticités prix croisés sont généralement très faibles, sinon statistiquement non différentes de zéro. Ces résultats renforcent d'autant plus notre hypothèse qu'ils sont obtenus sur des biens alimentaires. Pour lesquels la probabilité d'être substituables est supérieure aux classes agrégées de biens que nous utilisons ${ }^{8}$. La matrice de Slutsky s'écrit : $S_{b_{i}, b_{i}}^{h}$.

En substituant les expressions (10), (11) et (12) dans l'équation (7), puis en simplifiant, nous obtenons l'expression suivante de la condition du premier ordre de $t_{i}$ :

$$
\sum_{h} \Phi^{h}\left(x_{k}^{h}+\beta_{k} y_{k}^{h}\right)=\lambda\left[\sum_{h}\left(x_{k}^{h}+\beta_{k} y_{k}^{h}\right)+\sum_{i} \sum_{h}\left(t_{i} S_{b_{i}, b_{i}}^{h}\left(1+\beta_{i}^{2}\right)-t_{i}\left(x_{k}^{h} \frac{\partial x_{i}^{h}}{\partial I^{h}}+\beta_{i}^{2} y_{k}^{h} \frac{\partial y_{i}^{h}}{\partial I^{h}}\right)\right)\right]
$$

On pose à présent : $x_{k}=\sum_{h} x_{k}^{h}$ et $y_{k}=\sum_{h} y_{k}^{h}$, en substituant dans (13) et en simplifiant, nous obtenons :

$$
\frac{\sum_{h} t_{i} S_{b_{i}, b_{i}}^{h}\left(1+\beta_{i}^{2}\right)}{x_{i}+\beta_{i} y_{i}}=\left(\frac{\sum_{h}\left(\frac{\Phi^{h}}{\lambda}\right)\left(x_{i}^{h}+\beta_{i} y_{i}^{h}\right)}{x_{i}+\beta_{k} y_{i}}-1\right)+\frac{\sum_{i} \sum_{h} t_{i}\left(x_{k}^{h} \frac{\partial x_{i}^{h}}{\partial I^{h}}+\beta_{i}^{2} y_{k}^{h} \frac{\partial y_{i}^{h}}{\partial I^{h}}\right)}{x_{k}+\beta_{k} y_{k}}
$$

\footnotetext{
${ }^{8}$. On peut encore citer Deaton et Grimard (1993, p.18) : « None of these cross-price effects, except possibly that between wheat and rice, are large enough, or precise enough, to change by itself, the way in wich we might think about tax ».
} 
L'équation (14) fournit une expression de la solution optimale locale du taux de taxation proche de la formulation de Ramsey (1927) avec plusieurs agents. Les relations entre la solution des $t_{i}$ et les autres variables sont les suivantes :

2.2.1. Relation entre $t_{i}$ et la dérivée de la demande compensée aux prix $\left(S_{b_{i}, b_{i}}^{h}\right)$

Le terme de gauche de l'équation (14) représente un critère d'efficacité de la fiscalité. Il est de signe négatif. On observe que ce terme implique que les dérivées de la demande par rapport aux prix compensés (matrice de Slutsky) d'un bien i jouent négativement sur le taux de taxation optimal de ce bien. En cas de fortes élasticités prix, ou lorsque le bien est principalement consommé par les populations riches, les dérivées prix sont élevées. Dans ce cas, la réduction du taux de taxation permet de minimiser le poids mort produit par la taxation des biens. Autrement dit, on retrouve le résultat standard de la théorie de la fiscalité optimale qui est que le taux de taxation optimal d'un bien est une fonction décroissante de la dérivée de sa demande compensée par rapport aux prix. Ce critère est donc régressif du point de vue de la redistribution verticale.

2.2.2. Relation entre ti et la dérivée de la demande au revenu.

Le deuxième terme de droite de l'équation (14) $\sum_{i} \sum_{h} t_{i}\left(x_{k}^{h} \frac{\partial x_{i}^{h}}{\partial I^{h}}+\beta_{i}{ }^{2} y_{k}^{h} \frac{\partial y_{i}^{h}}{\partial I^{h}}\right)$ représente l'évolution des recettes fiscales en fonction de la sensibilité de la consommation à la variation des revenus. Ce terme est de signe négatif lorsque les revenus diminuent et sous l'hypothèse que les biens sont «normaux ». Autrement dit, la taxation provoque une diminution des revenus $\mathrm{I}^{\mathrm{h}}$ qui entraîne une réduction de la consommation des biens. Si la dérivée de la consommation du bien i par rapport au revenu est forte, alors la consommation diminuera de manière substantielle lorsque les impôts s'élèveront. L'efficacité de la collecte des impôts sera amenuisée par la réduction de l'assiette fiscale. Pour cette raison, le taux de taxation du bien i doit être alors relativement faible pour les biens dont la dérivée de la demande par rapport au revenu est forte, sous l'hypothèse que ces biens sont normaux. La dérivée de la demande par rapport au revenu étant a priori plus forte pour les individus «pauvres », cette dernière relation devrait favoriser la progressivité du système de taxation.

\subsubsection{Relation entre $t_{i}$ et le poids social des ménages}

Le premier terme de droite de l'équation (14) représente le critère d'équité dans la solution optimale de $t_{i}$. C'est une somme de la consommation taxée des ménages pondérée par le poids social de chaque ménage, moins 1. Il est généralement admis dans la littérature que ce terme est de signe négatif. Par ailleurs, on peut exprimer ce terme d'une manière plus explicite en suivant la méthode proposée par Feldstein (1972) :

$$
\sum_{h}\left(\frac{\Phi^{h}}{\lambda}\right)\left(\frac{x_{k}^{h}+\beta_{k} y_{k}^{h}}{x_{k}+\beta_{k} y_{k}}\right)=\operatorname{cov}\left(\frac{\Phi^{h}}{\lambda}, \frac{x_{k}^{h}+\beta_{k} y_{k}^{h}}{x_{k}+\beta_{k} y_{k}}\right)+\frac{1}{H} \sum_{h}\left(\frac{\Phi^{h}}{\lambda}\right)
$$


L'expression $\frac{1}{H} \sum_{h}\left(\frac{\Phi^{h}}{\lambda}\right)$ représente l'utilité marginale sociale moyenne de la population'. De fait, lorsque la consommation d'un bien i est corrélée positivement avec l'utilité marginale sociale d'un individu, la valeur absolue de $\frac{\sum_{h}\left(\frac{\Phi^{h}}{\lambda}\right)\left(x_{k}^{h}+\beta_{k} y_{k}^{h}\right)}{x_{k}+\beta_{k} y_{k}}-1$ diminue, ce qui entraîne un taux de taxation relativement faible du bien i. Ainsi, le taux de taxation doit être faible pour les biens qui sont consommés principalement par les individus dont le poids social est important et les individus dont l'utilité marginale des revenus est forte (i.e. les individus à faible revenu). De fait, plus la fonction d'utilité sociale $\mathrm{W}$ est concave (i.e. le poids social des catégories pauvres de ménages est élevé), plus le taux de taxation est faible.

2.2.4. Relation entre $t_{i}$ et le taux de couverture du secteur informel $\beta_{i}$.

Un taux de couverture du secteur informel élevé (pour une solution optimale donnée de $\beta i$ ), permet de réduire le taux de taxation optimal. En effet, on observe dans l'équation (14) que $\beta_{i}$ est facteur des variables : $S_{b_{i}, b_{i}}^{h},\left(\frac{\Phi^{h}}{\lambda}\right) \frac{\partial y_{i}^{h}}{\partial I^{h}}$, qui ont toutes une influence négative sur le taux de taxation optimal $\left(t_{i}\right)$. Ce résultat semble logique, puisqu'il indique que le taux de taxation normal appliqué à l'économie formelle pourra être réduit par une baisse de l'évasion fiscale du secteur informel, à recettes fiscales inchangées.

\subsection{La solution optimale (locale) de $\beta_{i}$}

La condition du premier ordre par rapport à $\beta_{\mathbf{i}}$ du programme de maximisation sous contrainte de l'Etat s'écrit :

$$
\frac{\partial L}{\partial \beta_{i}}=0 \Leftrightarrow \sum_{h}\left(\frac{\partial W}{\partial V^{h}} \frac{\partial V^{h}}{\partial q_{y_{i}}} \frac{\partial q_{y_{i}}}{\partial \beta_{i}}\right)=-\lambda\left[\sum_{h} t_{i} y_{i}^{h}+\sum_{i} \sum_{h}\left(t_{i} \frac{\partial q_{y_{i}}}{\partial \beta_{i}} \frac{\partial x_{i}^{h}}{\partial q_{y_{k}}}+\beta_{i} t_{i} \frac{\partial q_{y_{i}}}{\partial \beta_{i}} \frac{\partial y_{i}^{h}}{\partial q_{y_{k}}}\right)-g_{\beta_{i}}^{\prime}\right]
$$

En utilisant l'identité de Roy et en réutilisant les variables définies précédemment, on obtient :

$$
\sum_{h} \Phi^{h} y_{i}^{h} t_{i}=\lambda\left[\sum_{h} t_{i} y_{i}^{h}+\sum_{i} \sum_{h} t_{i}^{2}\left(\frac{\partial x_{i}^{h}}{\partial q_{y_{k}}}+\beta_{i} \frac{\partial y_{i}^{h}}{\partial q_{y_{k}}}\right)-g_{\beta_{i}}^{\prime}\right]
$$

Par hypothèse, les biens ne sont substituables qu'entre bien formel et informel (à l'intérieur d'une même paire de biens i). En réarrangeant l'équation (17), nous obtenons l'expression suivante de la solution de $\beta_{\mathrm{i}}$ :

$$
\beta_{i} t_{i} \sum_{h} \frac{\partial y_{i}^{h}}{\partial q_{y_{i}}}=\sum_{h}\left(\frac{\Phi^{h}}{\lambda}-1\right) y_{i}^{h}+g_{\beta_{i}}^{\prime}-t_{i} \sum_{h} \frac{\partial x_{i}^{h}}{\partial q_{y_{i}}}
$$

\footnotetext{
${ }^{9}$ On ne tient pas compte de l'interprétation qui pourrait être faite de $\lambda$. On notera toutefois qu'en cas de variation minime de $\mathrm{R}$ (les recettes fiscales), alors d'après la formulation du lagrangien, on peut écrire $: \lambda=-\partial \mathrm{W} / \partial \mathrm{t}_{\mathrm{i}} . \partial \mathrm{t}_{\mathrm{i}} / \partial \mathrm{R}$. D'après Ahmad et Stern (1991), $\lambda$ peut être alors interprété comme l'utilité marginale sociale du revenu du gouvernement, c'est à dire «le prix implicite des dépenses publiques » (traduction de shadow price).
} 


\subsubsection{Commentaires, cas d'une taxation optimale du secteur informel}

Si les biens informels sont des biens typiques, alors le terme de gauche de l'équation (18) sera de signe négatif. Supposons tout d'abord que le terme de droite soit aussi de signe négatif, de sorte que le taux de couverture du secteur informel soit positif (dans le cas contraire, la solution optimale pour l'Etat serait alors de subventionner le secteur informel). Sous ces hypothèses, la solution optimale de $\beta_{\mathrm{i}}$ dépend de ses arguments de la manière suivante :

Le terme de gauche $\sum_{h} \frac{\partial y_{i}^{h}}{\partial q_{y_{i}}}$ représente un critère d'efficacité du système de taxation. Il indique par exemple qu'une forte élasticité prix de la demande de bien informel doit jouer à la baisse sur le taux de couverture du secteur informel.

Les termes de droite impliquent:

$\beta_{\mathrm{i}}$ est une fonction décroissante de l'utilité sociale marginale du ménage $\left(\sum_{h} \frac{\Phi^{h}}{\lambda}\right)$. Autrement dit, le critère d'équité veut que le taux de taxation des biens informels soit réduit si ces biens sont d'avantages consommés par les ménages pauvres, et inversement.

Pour une raison d'efficacité du système, une forte convexité $\left(g_{\beta_{i}}^{\prime}\right.$ croissant) du coût de la taxation du secteur informel réduit le taux de couverture optimal du secteur informel. En effet une forte convexité du coût de fiscalisation du secteur informel (poids mort de la fraude) se traduit par un accroissement des coûts pour l'état, supérieur à celui de l'assiette fiscale. Par ailleurs, on notera que, sous cette hypothèse de convexité de la fonction de coût de fiscalisation du secteur informel, il est alors impossible que le taux de couverture optimal du secteur informel soit égal à 1 (si $\beta \rightarrow 1$ alors g' $\rightarrow \infty$ ). Ainsi, le taux de taxation du secteur informel est inférieur à celui du secteur formel, ce qui permet de réduire la charge supplémentaire (i.e. le poids mort) liée à la taxation de ce secteur $^{10}$.

En outre, une forte substituabilité entre les biens formels et informels $\left(\frac{\partial x_{i}^{h}}{\partial q_{y_{i}}}>0\right)$ influencera positivement le taux de couverture optimal du secteur informel. Il s'agit là aussi d'un critère d'efficacité. Dans l'hypothèse d'une forte substituabilité entre les deux types de biens, les incitations à la fraude fiscale sont d'autant plus importantes. Dans ce cas, l'écart entre les taux de taxation des deux types de biens (égal à $1-\beta_{\mathrm{i}}$ ) provoque une réduction de la base fiscale. La consommation des biens formels est substituée par celle des biens informels.

2.3.2. Cas où il est optimal de subventionner le secteur informel

Sous l'hypothèse standard que le bien informel est un bien normal et typique, le secteur informel doit être taxé (le terme de droite de l'équation 18 est positif, donc $\beta_{i} t_{i}$ l'est aussi) sous deux conditions :

- Le coût de taxation du secteur informel ne croît pas trop vite par rapport au nombre d'entreprises assujetties.

\footnotetext{
${ }^{10}$ On retrouve ainsi pour les mêmes raisons les résultats des modèles cités plus haut sur la fiscalité optimale avec fraude (Kaplow 1990, Cremer et Gahvary 1993).
} 
- Par ailleurs, la part des individus «non pauvres » consommant des biens informels doit être suffisamment importante (étant donné la concavité de la fonction d'utilité sociale).

Dans le cas contraire (terme de droite de l'équation (18) négatif), il serait donc optimal pour l'état de subventionner le secteur informel ( $\beta_{i} t_{i}$ négatif).

Considérons à présent le cas où le bien informel s'avérerait être un bien «Giffen ». La qualité du bien informel est très faible, lors d'une augmentation des prix du bien informel, l'effet revenu l'emporte, accroissant ainsi sa consommation.

Dans ce cas, $\sum_{h} \frac{\partial y_{i}^{h}}{\partial q_{y_{i}}}$ est positif. A supposer que le terme de droite de l'équation (18) reste de signe négatif, $\beta_{\mathrm{i}}$ est alors de signe négatif. Le secteur informel reçoit alors une subvention. Les commentaires sont alors les suivants :

- La relation entre $\beta_{\mathrm{i}}$ (en valeur absolue) et les termes de droite de l'équation (18) restent les mêmes par rapport aux variables $\sum_{h} \frac{\Phi^{h}}{\lambda}$ et $\mathrm{g}^{\prime} \beta$.

- En revanche, sous l'effet de la contrainte budgétaire, le signe de la dérivée entre la demande du bien formel et le prix du bien informel devient négatif. La hausse des dépenses en biens informels doit être compensée par une réduction de la consommation des biens formels, les biens deviennent ainsi complémentaires ${ }^{11}$. Dans ce cas, la sensibilité de la demande des biens formels au prix informel diminue la subvention optimale accordée au secteur informel.

En résumé, lorsque le bien informel est un bien typique, celui ci doit être taxé s'il est consommé de manière non marginale par les classes non défavorisées de la population, et si la convexité du coût lié à la fiscalisation du secteur informel n'est pas «trop » forte. Dans le cas contraire, le modèle prévoit de subventionner le secteur informel (empiriquement : à ne pas le taxer).

\section{MODELE EMPIRIQUE ET SIMULATIONS}

\subsection{Le modèle empirique}

Le modèle empirique doit permettre de fournir des estimations numériques des solutions optimales des taux de taxation et des taux de couverture du secteur informel. Pour cela, nous devons dans un premier temps choisir des formes fonctionnelles des fonctions d'utilité sociale et de demande.

$11 \frac{\partial x_{i}^{h}}{\partial q_{y_{i}}}<0$ 


\subsubsection{Poids social des ménages}

De manière standard, nous avons choisi de représenter la fonction d'utilité sociale par la fonction d'Atkinson (1970). Celle-ci s'écrit :

$$
W\left(V^{h}\right)= \begin{cases}\sum_{h} K \frac{\left(V^{h}\right)^{(1-\varepsilon)}}{(1-\varepsilon)} & \text { pour } \varepsilon \neq 1 \\ \sum_{\mathrm{h}} \mathrm{K} \cdot \ln \left(\mathrm{V}^{\mathrm{h}}\right) & \text { pour } \varepsilon=1\end{cases}
$$

L'exposant $\varepsilon$ représente l'élasticité de l'utilité sociale par rapport à l'utilité individuelle. C'est le «degré d'aversion aux inégalités de la société ». K est un facteur positif quelconque. Etant donné les caractéristiques de la fonction d'utilité sociale d'Atkinson (1970), on peut alors écrire $\Phi^{h}$ :

$$
\Phi^{h}=K\left[V^{h}\right]^{-\varepsilon} \frac{\partial V^{h}}{\partial I^{h}}
$$

Plus $\varepsilon$ est élevé, et moins l'Etat accordera d'importance au bien-être des ménages les plus aisés, et inversement. Autrement dit, plus $\varepsilon$ est grand, plus la société éprouve de l'aversion pour les inégalités. On rappelle que les caractéristiques de la fonction d'utilité collective d'Atkinson (1970) sont les suivantes : Pour $\varepsilon=0$, la société est «utilitariste»(Bentham): chaque individu est considéré de la même manière, quel que soit son revenu. Pour $\varepsilon \rightarrow \infty$, la société est «égalitariste » (Rawls) : l'utilité collective est égale à l'utilité de l'individu le plus défavorisé de la société.

Nous choisissons une forme PIGLOG pour représenter la fonction d'utilité indirecte, qui permet ensuite d'être cohérent avec le choix d'une fonction AIDS pour modéliser la demande des consommateurs (cf. infra) :

$$
\ln V^{h}\left(I^{h}, p\right)=\frac{\ln \left(I^{h} / n^{h}\right)-\ln a^{h}(p)}{b^{h}(p)}
$$

Où $\mathrm{a}^{\mathrm{h}}(\mathrm{p})$ et $\mathrm{b}^{\mathrm{h}}(\mathrm{p})$ représentent des indices de prix du consommateur reflétant ses préférences (Cf. infra). La variable $\mathrm{n}^{\mathrm{h}}$ est le nombre d'équivalent adulte dans le ménage $\mathrm{h}$. L'échelle d'équivalence retenue est celle de l'OCDE (poids de 1 pour le chef de ménage, 0,7 pour les autres adultes et 0,5 pour les enfants - on définit un adulte comme étant une personne de moins de 16 ans).

Afin de simplifier $\Phi^{h}$, on transforme cette expression de sorte à la normaliser à 1 pour le consommateur le plus démuni. On supprime de plus le rapport des utilités marginales du revenu. L'approximation du poids social des individus est alors :

$$
\Phi^{h}=\left[\frac{v^{1}}{v^{h}}\right]^{\varepsilon} \quad \text { où v } v^{1} \text { est l'utilité de l'individu le plus pauvre. }
$$

3.1.2. Formes fonctionnelles des solutions de $t_{i}, \beta_{i}$ et $\lambda$

Les revenus compensés ne peuvent pas être observés dans la réalité. Cela implique que les élasticités prix sont estimées à partir des variations totales de la demande, comprenant l'effet substitution et l'effet revenu. Autrement dit, le programme de fiscalité optimale ne peut pas intégrer l'équation de Slutsky de manière empirique. Par ailleurs, ne disposant pas d'informations sur le surcoût lié à la fiscalisation du secteur informel, nous préférons ne pas en tenir compte dans 
l'estimation empirique du système fiscal optimal. Cette simplification aura bien sûr pour effet d'augmenter le taux de taxation optimal des biens informels. De plus, nous maintenons l'hypothèse d'élasticités prix croisés non nulles uniquement entre les biens formels et informels appartenant à la famille de bien $i$.

La solution optimale de $t_{i}$ est alors représentée par la fonction suivante :

$$
\sum_{h} \Phi^{h}\left(x_{k}^{h}+\beta_{k} y_{k}^{h}\right)=\lambda\left[\sum_{h}\left(x_{k}^{h}+\beta_{k} y_{k}^{h}\right)+t_{i} \sum_{h}\left(\frac{\partial x_{i}^{h}}{\partial q_{x_{i}}}+\frac{\partial x_{i}^{h}}{\partial q_{y_{i}}}+\beta_{i}^{2}\left(\frac{\partial y_{i}^{h}}{\partial q_{y_{i}}}+\frac{\partial y_{i}^{h}}{\partial q_{x_{i}}}\right)\right)\right]
$$

Celle de $\beta_{i}$ est donnée par l'équation (17):

$$
\sum_{h} \Phi^{h} y_{i}^{h}=\lambda\left[\sum_{h} y_{i}^{h}+t_{i} \sum_{h} \cdot\left(\frac{\partial x_{i}^{h}}{\partial q_{y_{i}}}+\beta_{i} \frac{\partial y_{i}^{h}}{\partial q_{y_{i}}}\right)\right]
$$

La contrainte de l'Etat reste :

$$
R_{0}=\sum_{i=1}^{n} \sum_{h=1}^{H}\left(t_{i} x_{i}^{h}+\beta_{i} t_{i} y_{i}^{h}\right)
$$

Les équations (23) à (25) permettent alors de définir un système juste identifié de $2 \mathrm{n}+1$ équations, permettant d'obtenir les solutions des $n . t_{i}, n . \beta_{i}$. La variable $\Phi^{h}$ est calculée grâce à l'équation (22).

\subsection{Les fonctions de demande de biens}

Les fonctions de demande vont permettre de prendre en compte les variations de la demande de biens induites par la variation des taxes. La forme retenue est une fonction AIDS Deaton et Muellbauer 1980). Son choix présente un certain nombre d'avantages par rapport à d'autres formes de fonction de demande couramment utilisées telles que la LES (Linear Expenditure System) ou la CES (Constant Elasticity Substitution) ${ }^{12}$ :

- L'AIDS permet d'obtenir des courbes d'Engel qui ne sont pas linéaires. Le fait que les préférences ne soient pas homothétiques est important car, dans le cas contraire, la théorie montre (Atkinson 1977) qu'un taux de TVA uniforme est optimal. De plus, on peut remarquer qu'il existe alors une incohérence lorsque l'on estime les élasticités à partir d'une fonction LES ou CES et que l'on calibre le modèle à partir de données d'enquêtes où la part budgétaire des biens pour chaque ménage n'est pas constante.

- En outre, les biens sont forcément complémentaires pour une LES. A l'inverse, la fonction de demande AIDS permet de prendre en compte des biens substituables, et donc d'étudier les effets de la substituabilité entre les biens informels et formels.

La demande pour un bien i par le ménage h s'écrit :

$$
w_{b i}^{h}=\alpha_{b i}^{h}+\sum_{k} d_{b_{i}, b i}^{h} \ln q_{b k}+c_{b i}^{h} \ln \frac{\left(I^{h} / n^{h}\right)}{Q}
$$

\footnotetext{
${ }^{12}$ D'un point de vue pratique, des fonctions AIDS ont déjà été estimées dans de nombreux pays en développement, dont Madagascar (Ravelosoa et al. 1999).
} 
Où $\alpha_{b i}^{h}, d_{b i, b i}^{h}$ et $c_{b i}^{h}$ sont les paramètres à estimer. $w_{b i}^{h}$ est la part budgétaire du bien $b_{\text {i }}$ pour le ménage $\mathrm{h}$, q $\mathrm{bk}$ la matrice des prix au consommateur, $\mathrm{I}^{\mathrm{h}}$ la dépense totale du ménage ${ }^{13}$. Enfin, $\mathrm{Q}$ est l'indice des prix à la consommation, estimé par l'indice de Stone :

$$
\ln Q=\sum_{k} w_{b k} \ln q_{b k} \quad \text { avec } w_{b k}=\sum_{h} \frac{q_{b k} b_{b k}^{h}}{I^{h}}
$$

Les paramètres de la fonction d'utilité indirecte du consommateur sont alors :

$$
\ln a^{h}(p)=\sum_{k} w_{b k}^{h} \ln q_{b k} \quad \text { et } \quad \ln b^{h}(p)=\prod_{k} p_{k}^{c_{b i}^{h}}
$$

\subsubsection{Résolution du système}

Le programme de l'Etat est représenté par un système de $2 n+1$ équations. En parallèle, le système de demande des consommateurs est composé de $2 \mathrm{n}$ équations (demande de biens formels et informels). Au total, le modèle est donc constitué de $4 n+1$ équations.

A notre connaissance, c'est la première fois qu'un modèle empirique de fiscalité optimale est résolu en tenant compte explicitement des variations de la demande entraînées par celles des taux de taxation (voir Ray 1997 pour une revue). Or, les variations des taux de taxation, et donc des prix, entraînées par le calcul des taxes optimales, ne sont pas toujours «marginales ». Les résultats des modèles risquent alors d'être fortement biaisés sous l'hypothèse de demandes constantes.

De manière pratique, le système d'équations (21) à (28), plus les équations de prix au consommateur, sont retranscrits sous le logiciel GAMS. Ceci permet ensuite de déterminer de manière simultanée : les taux de taxation optimaux, le taux de couverture optimale du secteur informel, la demande de biens des ménages et les prix au consommateur. Les élasticités et les dérivées de la demande par rapport aux prix et revenu, ainsi que les paramètres de la fonction AIDS, sont en revanche supposés exogènes, afin de faciliter la résolution du modèle.

Nos simulations consistent ensuite à faire varier les indices d'aversion aux inégalités pour montrer quel serait le système de taxation des biens formels et informels qui serait dans ce cas le plus approprié.

\subsubsection{Paramétrage du modèle.}

Etant donné la taille de l'enquête ménage (400 ménages) et de l'unité géographique de l'enquête MADIO (1995b), il est peu aisé d'estimer des systèmes de demande de type AIDS permettant de calculer les élasticités prix requises pour le paramétrage du modèle. La méthode proposée par Deaton (1997) pour estimer les élasticités prix à partir de la variance spatiale des prix est ici difficilement applicable du fait de l'unité spatiale de l'enquête. Ainsi, dans cette enquête, la variance des prix d'un bien proviendra probablement en grande partie des différences de qualités entre les produits. Le risque est alors d'estimer des comportements de choix de qualité, au lieu de comportements de consommation en fonction des prix (Deaton 1997). Contrôler le choix de la qualité accroît encore la complexité la méthode. Pour ces raisons, nous avons choisi d'utiliser des paramètres déjà calculés dans des études antérieures : Pour les produits alimentaires, Ravelosoa

\footnotetext{
${ }^{13}$ Calculer la fonction de demande par rapport au revenu équivalent $\left(\mathrm{I}^{\mathrm{h}} / \mathrm{n}^{\mathrm{h}}\right)$ permet ensuite de calculer une agrégation exacte des demandes individuelles (Cf. Deaton et Muellbauer 1980). Les simulations retenues ont été en effet effectuées sur des classes agrégées de ménages (cf. infra).
} 
et al. (1999) ont déjà estimé des fonctions de demande de type AIDS à Madagascar. Il existe en revanche peu de résultats pour les produits autres qu'alimentaires dans les pays en développement. Seul Goaïed (1991) nous fournit une estimation des élasticités prix et revenu du logement en Tunisie. Pour les autres biens, les élasticités sont tirées du travail de Nichèle et Robin (1993) pour la France. Ces élasticités sont présentées dans le tableau 3-1.

Toutefois, ces études ont estimé les élasticités prix et revenus des produits de manière indifférenciée entre les biens formels et informels. Nous sommes alors obligés d'émettre des hypothèses sur la valeur des paramètres entre les biens formels et informels. Nous nous appuyons pour cela sur les résultats de l'enquête MADIO (1995b), et les travaux de Roubaud (1994) et Cogneau et al. (1996) au Cameroun ${ }^{14}$ :

- Le tableau 1-2 (supra) montre que la part budgétaire des biens et services informels décroît avec le revenu des ménages. Les biens informels seraient donc des biens nécessaires (élasticité revenu inférieure à 1), tandis que les biens formels seraient des biens de luxe (voir aussi Roubaud 1994 ou Cogneau et al. 1996 pour le Cameroun). L'exemple de la consommation du poste «Riz et céréales » est assez parlant de ce point de vue. On observe en effet dans l'enquête que la totalité du riz ordinaire et paddy consommée provient du secteur informel, lorsque le riz de luxe est en revanche entièrement acheté dans le secteur formel. Ainsi, à l'image de Cogneau et al. (1996), on peut supposer que les élasticités revenu des biens informels sont inférieures à celles des biens formels, voire inférieures à 1 .

- L'enquête auprès des ménages MADIO (1995b, p.20-21) fait ressortir que le secteur informel est choisi pour la modicité des prix, tandis que les biens formels sont choisis pour leur meilleure qualité. L'élasticité prix des biens informels doit être alors inférieure en valeur absolue à celle des biens formels (Cogneau et al. 1996). N'étant pas en mesure d'estimer les élasticités de la consommation, nous écartons le cas où les biens informels seraient des biens «Giffen».

Les élasticités du tableau 3-1 représentent des valeurs moyennes sur l'ensemble des biens. Pour les raisons exposées ci-dessus, les élasticités des biens formels et informels ont été fixées arbitrairement de la manière suivante : les élasticités revenu (prix directs) du tableau 3-1 sont augmentées (diminuées) de $10 \%$ pour les biens formels et diminuées (augmentées) de $5 \%$ pour les biens informels.

Tableau $n^{\circ}$ 3-1 : Elasticités prix et revenus et distribution de la demande

\begin{tabular}{|l|c|c|c|c|c|}
\hline Biens et services & $\begin{array}{c}\text { Elasticité } \\
\text { revenu }\end{array}$ & $\begin{array}{c}\text { Elasticité prix } \\
\text { directe }\end{array}$ & $\begin{array}{c}\text { Elasticité prix } \\
\text { croisée }\end{array}$ & $\begin{array}{c}\text { Distribution des } \\
\text { biens informels }\end{array}$ & $\begin{array}{c}\text { Distribution des } \\
\text { biens formels }\end{array}$ \\
\hline Riz, céréales (1) & 0.20 & -0.70 & 0.52 & $25.3 \%$ & $0.9 \%$ \\
Viandes, légumes, (1) & 1.30 & -0.85 & 0.70 & $30.6 \%$ & $1.4 \%$ \\
Produits transformés (1) & 1.40 & -0.87 & 0.70 & $8.0 \%$ & $19.8 \%$ \\
Habillement (2) & 1.17 & -0.91 & 0.78 & $3.3 \%$ & $13.2 \%$ \\
Logement (3) & 1.17 & -1.47 & 0.86 & $13.6 \%$ & $12.4 \%$ \\
Equipement maison, (3) & 1.17 & -1.47 & 0.90 & $4.0 \%$ & $7.6 \%$ \\
Santé (2) & 1.09 & -1.00 & 0.62 & $1.0 \%$ & $10.8 \%$ \\
Transport (4) & 1.30 & -1.15 & 0.90 & $10.2 \%$ & $24.6 \%$ \\
Education (2) & 1.17 & -0.58 & 0.50 & $2.4 \%$ & $5.9 \%$ \\
Autres biens et services (4) & 1.20 & -1.10 & 0.88 & $1.8 \%$ & $3.4 \%$ \\
Total & & & & $100.0 \%$ & $100.0 \%$ \\
\hline
\end{tabular}

Sources : (1) Ravelosoa et al. (1999), (2) Nichèle et Robin (1993), (3)Goaïed (1991), (4) valeur arbitraire.

\footnotetext{
${ }^{14}$ Voir aussi Boyabé (1999) pour une revue.
} 
Enfin, il s'agit de choisir les élasticités prix croisées entre les biens informels et formels. Celles-ci sont en revanche clairement positives, les deux biens sont en effet substituables par hypothèse. Ils le sont toutefois imparfaitement du fait de la différence de qualité des produits. On peut supposer que cette imparfaite substituabilité entre les biens se reflète dans la part du bien informel dans la consommation totale d'un bien $\mathrm{i}^{15}$.

Une fois choisies les élasticités prix et revenus, on en déduit facilement la valeur des paramètres $d_{b i, b i}^{h}, c_{b i}^{h}$ et $d_{b i, b k}^{h}$ des fonctions de demande, à partir des formules des élasticités des fonctions AIDS $^{16}$. La constante de la fonction de demande est ensuite déterminée comme la différence entre les termes de gauche et de droite des équations de demande.

Par ailleurs, l'homogénéité de degré zéro et la «contrainte d'additivité » au sein d'une fonction de demande AIDS impliquent que : $\sum_{k} d_{b i, b k}^{h}=0, \sum_{i} c_{b i}^{h}=0, \sum_{\mathrm{i}} \mathrm{a}_{\mathrm{bi}}^{\mathrm{h}}=1$. Les paramètres n'étant pas estimés, il était alors difficile de les fixer de manière à satisfaire ces contraintes, sans obtenir ensuite des valeurs aberrantes des dérivées de la demande. La contrainte budgétaire est néanmoins respectée en calculant de manière résiduelle la demande des «autres biens et services » informels. Le modèle respecte aussi la symétrie de Slutsky $: d_{x_{i}, y_{i}}^{h}=d_{y_{i}, x_{i}}^{h}$.

\subsection{Résultats des simulations}

Le paramétrage du modèle nous a amené à émettre des hypothèses sur la typologie des biens informels. Celles-ci ne sont pas sans conséquences sur les résultats du modèle empirique. Il est alors utile de préciser ces liens :

- Les élasticités revenu relativement faibles d'une part, et la forte substituabilité entre les biens formels et informels d'autre part, doit accroître (selon les critères d'efficacité) le taux de taxation optimal des biens informels. Toutefois, les dérivées de la demande par rapport aux revenus devraient réduire la taxation optimale des biens informels, ceux-ci étant plus consommés par les ménages à bas revenus.

- En revanche, les préférences des catégories défavorisées pour les biens informels tendront à diminuer la pression fiscale sur ces biens (critère d'équité).

- Les dérivées de la demande par rapport aux prix (directes) ont des effets contradictoires sur le niveau de taxation des biens informels. D'une part, les élasticités prix des biens informels étant supposées plus importantes que celles des biens formels (pour un même bien i), le taux de taxation du bien informel doit être plus faible (efficacité). En revanche, les biens formels étant principalement consommés par les hauts revenus, les dérivées prix seront probablement plus importantes pour ces biens. Il est en fait plus probable que les dérivées prix (directes) jouent en faveur d'une réduction de la taxation des biens formels.

\footnotetext{
${ }^{15}$ Plus la part des deux biens dans la consommation totale est proche de $50 \%$, plus les biens sont a priori substituables. On choisit d'estimer les élasticités prix croisées à partir de la formule suivante : $1-\left|0.5-\frac{q_{y_{i}} y_{i}}{q_{y_{i}} y_{i}+q_{x_{i}} x_{i}}\right|$.

${ }^{16} e_{R}=1+\frac{c_{b i}^{h}}{w_{b i}^{h}}$ (élasticité revenu), $e_{i i}=-1+\frac{d_{b i, b i}^{h}}{w_{b i}^{h}}-c_{b i}^{h}$ (élasticité prix directe), et $e_{i k}=\frac{d_{b i, b k}^{h}}{w_{b i}^{h}}-\frac{c_{b i}^{h}}{w_{b i}^{h}} w_{b k}^{h}$ (élasticité prix croisée).
} 
Nos simulations permettent à présent d'estimer les taux de taxation optimaux selon le degré de redistribution verticale recherché par l'Etat au travers de son système d'imposition indirecte. Les simulations consistent en effet à calculer les effets des variations de la variable $\varepsilon$ sur les variables endogènes du modèle $(t, \beta, q)$.

Dans un premier temps nous avions calculé les taux de taxation optimaux à partir des 400 ménages de notre échantillon. Or les résultats produits de cette manière sont difficilement interprétables du fait d'une forte hétérogénéité des préférences intra-classes. On peut en effet observer dans le tableau 2 (en annexe) que les coefficients de variation des taux de pression intra-déciles sont nettement supérieurs à la variation des taux de pression moyens inter-déciles. Ceci témoigne alors d'une forte variation des préférences entre les individus de même revenu, plus importante que pour les individus ayant des revenus différents. Cette variance intra-déciles produit des mouvements erratiques des taux de taxation optimaux par produits lorsque les indices d'aversion aux inégalités varient. Pour limiter ces effets et «linéariser » nos résultats, nous avons alors calculé les taux de taxation optimaux à partir de classes de dépenses agrégées par vingtiles.

\subsubsection{Taxation des biens formels versus taxation des biens informels.}

La taxation relative des deux secteurs est fonction du degré de redistribution souhaité. L'accroissement du poids social des populations les plus défavorisées tend à accroître l'écart des taux de taxation entre les secteurs formels et informels. A l'opposée, les critères d'efficacité ont pour conséquence d'accroître le taux de pression sur les biens informels. In fine, l'écart entre les taux de taxation optimaux des deux secteurs est plus faible que celui existant dans le système actuel (cf. tableau 3-2). Ainsi, les taux de taxation optimaux moyens des biens et services formels sont inférieurs aux taux actuels (cf. tableau 3-2). A l'opposée, les produits informels subissent en moyenne un taux de pression plus élevé que le taux actuel, pour des valeurs des indices d'aversion de 0 ou de 1 .

En outre, la redistribution des taux de taxation entre les biens accroît la part budgétaire des biens formels (cf. tableau 1 en annexe). Ainsi, pour un indice d'aversion aux inégalités de 2 par exemple, le taux de taxation moyen du secteur formel est inférieur au taux actuel, bien que le taux de pression moyen des biens informels soit identique. Ainsi, si le taux de taxation optimal moyen du secteur informel reste peu élevé (entre $1,1 \%$ et $0,3 \%$ ), la hausse de la taxation de certains biens informels permet de réduire la pression moyenne du secteur formel. Cette évolution du système de taxation devrait alors occasionner un gain d'efficacité.

En conclusion, il semblerait optimal d'accroître le taux de pression sur certains produits informels. Pour un indice d'aversion aux inégalités de 1 , le taux de pression moyen des biens informels passerait à $1 \%$, contre un taux de $0,3 \%$ actuellement. A recettes constantes, le remaniement du système de taxation permettrait de réduire le taux de pression moyen des biens formels. Toujours pour un indice d'aversion aux inégalités de 1, le taux de pression optimal sur les biens formels serait $6,6 \%$, contre $7,6 \%$ actuellement.

On note que les taux optimaux moyens sur les secteurs informel et formel sont proches de l'état initial. Ce résultat tient au fait que l'algorithme de résolution employé par GAMS (CONOPT 2 et MINOS 5) est «influencé » par les équilibres de départ dans le sens où la solution des fonctions de demande tend à converger vers l'équilibre initial. Les variations possibles des taux de taxation sont ainsi limitées. Les résultats sont plus saillants si le modèle est résolu sous l'hypothèse d'une demande exogène. Si les valeurs ordinales sont similaires, les écarts entre les taux de pression entre secteurs formel et informel sont en revanche plus amples. Ainsi le taux de pression optimal du secteur informel est de $1,8 \%$ pour $\varepsilon=0$, et tombe à $-3,6 \%$ (subvention sur les prix des biens 
informels) pour $\varepsilon=3$. Si les variations avec demande endogène sont moins marquées, elles ont néanmoins l'avantage de mieux prendre en compte les effets substitutions (voir aussi Banks et al. 1996). Effets qui peuvent aussi expliquer la faiblesse des évolutions des taux lorsque la demande est endogène. De plus, cette évolution limitée des taux pour chaque produit permet aux résultats de nos simulations d'être directement une source possible de réformes fiscales. Ainsi, les résultats du modèle avec demande endogène sont plus riches d'enseignements du point de vue empirique.

\subsubsection{Résultats sur la taxation des biens et services}

Bien que les taux de taxation optimaux soit calculés à partir de dépenses agrégées, leurs mouvements restent parfois erratiques lorsque l'indice d'aversion aux inégalités varie. Toutefois, des résultats notables semblent ressortir des simulations (cf. tableau 3-2) :

Les taux de taxation optimaux des produits alimentaires divergent peu de ceux du système actuel. Nos simulations font toutefois ressortir qu'il serait optimal d'assurer un taux de taxation entre 6 et $8 \%{ }^{17}$ des produits alimentaires transformés, qu'ils soient d'origine formelle ou informelle. De même, pour les produits de l'élevage, de la pêche, les fruits et les légumes, il pourrait être optimal de taxer ces biens à des taux compris entre 5 et $7 \%$ pour des niveaux de progressivité élevés $(\varepsilon=1$ et $\varepsilon=2$ ). En revanche, les céréales, qui sont des biens nécessaires, sont taxées au taux zéro. Une réforme fiscale consisterait donc à assurer l'application d'un taux zéro sur l'ensemble des céréales. Appliquer un taux de $5 \%$ sur les produits alimentaires (hors produits de base) serait en revanche une réforme possible. On remarque aussi que la taxation des céréales, qui est actuellement due à une rémanence de la TVA pour les biens exonérés, est bien à l'origine d'effets régressifs.

L'habillement pourrait être plus amplement taxé pour des critères d'efficacité. Il est intéressant de noter que pour $\varepsilon=1$, le taux de taxation optimal sur l'habillement est proche du taux normal de la TVA. La réforme pour l'Etat consisterait alors à supprimer les exemptions accordées aux associations de sorte à appliquer de manière universelle le droit fiscal.

Nous constatons ensuite que la taxation optimale des secteurs sociaux : éducation, santé n'est pas négligeable. Un taux de pression d'environ $3 \%$ sur la consommation des services sociaux formels (soit un taux de l'ordre de $5 \%$ sur les prix au producteur) pourrait représenter une solution optimale. Ce résultat, a priori étonnant, n'est en fait pas illogique : L'éducation et la santé étant deux biens de luxe, dans le sens où leur part budgétaire croît plus vite que les revenus, et avec des élasticités-prix faibles, il est en fait logique d'obtenir un tel résultat dans un modèle de fiscalité optimale $^{18}$. On peut d'ailleurs s'interroger sur le fait qu'en exonérant les services sociaux de manière indifférenciée, on accorde un cadeau fiscal plus important aux couches les plus favorisées de la population. La solution pourrait être alors de taxer les services sociaux formels, puis de reverser le produit de ces taxes en subventionnant l'accès de ses services pour les populations les plus pauvres. On augmenterait ainsi la progressivité du système de taxes et de dépenses publiques. On notera par ailleurs, que les récentes propositions de réforme fiscale visant à taxer les produits pharmaceutiques à Madagascar (proposition de loi de finances 1999) vont dans le sens d'une fiscalité indirecte optimale. Sachant qu'il existe par ailleurs des centrales d'achat de médicaments où ceux-ci sont subventionnés.

Du point de vue pratique, il pourrait être optimal d'introduire des taux différenciés dans la taxation des biens de consommation à Madagascar. Un taux de $5 \%$ sur le prix au producteur des biens

\footnotetext{
${ }^{17}$ soit un taux d'environ $10 \%$ sur le prix au producteur.

${ }^{18}$ Prendre en compte les externalités et les effets à long terme créés par les secteurs sociaux limite bien sûr la pertinence d'un tel résultat.
} 
alimentaires (hors céréales), les services sociaux et le logement serait une réforme envisageable dans ce but. Les exonérations doivent être remplacées par un taux zéro pour les céréales. Les effets de rémanence pourraient être ainsi éliminés. Pour les autres produits, on notera que les taux maximum sont généralement inférieurs au taux normal actuel (soit un taux de 16,7 \% rapporté au prix au consommateur ou de $20 \%$ par rapport au prix au producteur). A recettes constantes, un abaissement du taux «normal» sur les prix au producteur autour de $15 \%$ serait envisageable, étant donné la taxation des biens alimentaires et des services sociaux au taux de $5 \%$. Cette réforme aurait de plus pour effet de réduire les incitations sur la fraude et donc de réduire les distorsions créées par la TVA sur le jeu de la concurrence. L'autre côté de la réforme consisterait à taxer le secteur informel de manière ciblée. Les simulations montrent que le taux de pression devrait être essentiellement accru sur les secteurs de l'«agro-industrie » et de l'habillement de manière à les rapprocher des taux des entreprises formelles. 
Tableau n $^{\circ}$ 3-2 : Taux de taxation optimaux avec demande endogène

\begin{tabular}{|c|c|c|c|c|c|}
\hline $\begin{array}{l}\text { Degré d'aversion aux inégalités } \\
\text { Taux de taxation des biens formels }\end{array}$ & Initial & $\varepsilon=0$ & $\varepsilon=1$ & $\varepsilon=2$ & $\varepsilon=3$ \\
\hline Riz, céréales & $2.7 \%$ & $1.2 \%$ & $0.0 \%$ & $0.3 \%$ & $1.8 \%$ \\
\hline Viandes, poisson, légumes, fruits... & $0.7 \%$ & $0.6 \%$ & $0.0 \%$ & $7.5 \%$ & $4.9 \%$ \\
\hline Alimentation transformée & $10.0 \%$ & $5.3 \%$ & $7.9 \%$ & $6.2 \%$ & $11.7 \%$ \\
\hline Habillement & $3.7 \%$ & $9.9 \%$ & $16.3 \%$ & $4.4 \%$ & $2.6 \%$ \\
\hline Logement & $3.8 \%$ & $1.8 \%$ & $4.8 \%$ & $2.5 \%$ & $4.3 \%$ \\
\hline Equipement, services maisons & $11.7 \%$ & $7.0 \%$ & $10.8 \%$ & $10.1 \%$ & $10.5 \%$ \\
\hline Santé & $1.5 \%$ & $13.6 \%$ & $3.1 \%$ & $3.4 \%$ & $1.6 \%$ \\
\hline Education & $1.1 \%$ & $6.5 \%$ & $2.0 \%$ & $1.8 \%$ & $15.9 \%$ \\
\hline Transport & $13.7 \%$ & $6.8 \%$ & $-0.1 \%$ & $12.8 \%$ & $8.4 \%$ \\
\hline Autres biens et services & $10.0 \%$ & $6.9 \%$ & $8.4 \%$ & $19.4 \%$ & $8.8 \%$ \\
\hline Moyen & $7.8 \%$ & $7.0 \%$ & $6.6 \%$ & $7.1 \%$ & $7.6 \%$ \\
\hline \multicolumn{6}{|l|}{ Taux de taxation des biens informels } \\
\hline Riz, céréales & $0.0 \%$ & $0.1 \%$ & $0.0 \%$ & $0.0 \%$ & $0.2 \%$ \\
\hline Viandes, poisson, légumes, fruits... & $0.0 \%$ & $0.1 \%$ & $0.0 \%$ & $-0.4 \%$ & $0.4 \%$ \\
\hline Alimentation transformée & $1.1 \%$ & $-0.7 \%$ & $8.3 \%$ & $6.0 \%$ & $2.3 \%$ \\
\hline Habillement & $1.2 \%$ & $20.8 \%$ & $1.3 \%$ & $0.5 \%$ & $0.8 \%$ \\
\hline Logement & $0.5 \%$ & $0.3 \%$ & $0.8 \%$ & $0.3 \%$ & $0.7 \%$ \\
\hline Equipement, services maisons & $1.5 \%$ & $5.0 \%$ & $2.8 \%$ & $-2.5 \%$ & $1.8 \%$ \\
\hline Santé & $0.0 \%$ & $40.7 \%$ & $-1.2 \%$ & $0.2 \%$ & $0.0 \%$ \\
\hline Education & $0.0 \%$ & $9.8 \%$ & $-0.3 \%$ & $-0.4 \%$ & $-0.7 \%$ \\
\hline Transport & $0.0 \%$ & $-9.9 \%$ & $0.1 \%$ & $0.0 \%$ & $-1.8 \%$ \\
\hline Autres biens et services & $0.3 \%$ & $5.6 \%$ & $-1.1 \%$ & $-0.1 \%$ & $-0.1 \%$ \\
\hline Moyen & $0.3 \%$ & $1.1 \%$ & $1.0 \%$ & $0.3 \%$ & $0.3 \%$ \\
\hline \multicolumn{6}{|l|}{ Structure des recettes fiscales } \\
\hline Riz, céréales & $0.3 \%$ & $0.6 \%$ & $0.0 \%$ & $0.0 \%$ & $1.0 \%$ \\
\hline Viandes, poisson, légumes, fruits... & $0.4 \%$ & $0.6 \%$ & $0.0 \%$ & $-1.0 \%$ & $2.9 \%$ \\
\hline Alimentation transformée & $25.8 \%$ & $11.4 \%$ & $32.1 \%$ & $22.9 \%$ & $33.8 \%$ \\
\hline Habillement & $7.1 \%$ & $31.1 \%$ & $33.5 \%$ & $9.6 \%$ & $5.3 \%$ \\
\hline Logement & $7.6 \%$ & $3.5 \%$ & $11.9 \%$ & $4.5 \%$ & $8.4 \%$ \\
\hline Equipement, services maisons & $11.9 \%$ & $10.4 \%$ & $12.5 \%$ & $9.4 \%$ & $10.6 \%$ \\
\hline Santé & $2.1 \%$ & $27.8 \%$ & $4.7 \%$ & $5.7 \%$ & $2.2 \%$ \\
\hline Education & $0.9 \%$ & $9.8 \%$ & $1.6 \%$ & $1.8 \%$ & $10.4 \%$ \\
\hline Transport & $39.6 \%$ & $-0.1 \%$ & $-0.1 \%$ & $44.3 \%$ & $21.6 \%$ \\
\hline Autres biens et services & $4.3 \%$ & $4.9 \%$ & $4.1 \%$ & $2.9 \%$ & $3.9 \%$ \\
\hline Total & $100.0 \%$ & $100.0 \%$ & $100.0 \%$ & $100.0 \%$ & $100.0 \%$ \\
\hline
\end{tabular}

\section{CONCLUSION}

Les préférences des consommateurs «pauvres » pour les biens informels et l'élasticité prix élevée de ces biens justifie leur faible taxation. A partir d'un modèle de fiscalité optimale, nous estimons en effet que les taux de taxation des biens informels sont nettement inférieurs à ceux des biens formels.

Si nos résultats sont globalement proches de ceux obtenus par les modèles analytiques de fiscalité optimale avec fraude (Kaplow 1990, Cremer et Gahvary 1993), il existe toutefois des nuances importantes dans les raisons d'y parvenir. Dans notre modèle, la faiblesse du taux de taxation des biens informels ne provient pas d'un surcoût lié à la taxation du secteur frauduleux, mais comme nous l'avons vu plus haut de la typologie des biens. Ensuite le fait que la taxation du bien informel 
soit inférieure à celle du bien formel n'est pas vérifié pour tous les secteurs de l'économie, contrairement à ce qui est conclu par les modèles analytiques.

L'introduction dans le modèle empirique d'un surcoût lié à la taxation du secteur informel aurait encore accru l'écart des taux de taxation entre les secteurs formels et informels (réduction du poids mort lié à la taxation de la fraude). Le surcoût lié à la fiscalisation de l'informel ne peut être connu que si l'on dispose de statistiques sur les coûts de fonctionnement de l'administration. Information qui n'est généralement pas disponible dans les PED.

En revanche, la modélisation en équilibre général est envisageable. Celle-ci permettrait de tenir compte de deux effets importants. Elle favoriserait d'une part un accroissement de la taxation du secteur informel. En effet, tout écart des taux de taxation entre les secteurs se traduirait par une réallocation des facteurs de production des secteurs formels vers l'informel. Ceci affecterait la contrainte budgétaire de l'Etat, encourageant ainsi la taxation du secteur informel. A l'opposée, la taxation des biens informels doit réduire le prix au producteur informel, et donc le revenu des ménages les moins favorisés. Il serait donc intéressant de développer ce modèle dans un EGC, sachant que nous avons déjà estimé la probabilité pour une entreprise d'être formelle ou informelle en fonction des taux de taxation. Par ailleurs, Fortin et al. (1997) ont déjà endogénéisé le secteur informel dans un modèle EGC. 


\section{REFERENCES}

Ahmad E. et Stern N. (1991), «The theory and practice of tax reform in developing countries », Cambridge University Press.

Atkinson A.B. (1970), «On the measurement of inequality», Journal of Economic Theory, vol 2, pp. 244-63.

Boyabé J.B. (1999), « Marché informel : une lecture critique du modèle d'Akerlof », Revue Tiers Monde, tome XL, ${ }^{\circ} 157$, janvier-mars, pp. $169-186$.

Banks J., Blundell R. et Lewbel R. (1996), «Tax reform and welfare measurement : do we need demand system estimation ? », The Economic Journal, 106, pp. 1227-41.

Banks J., Blundell R. et Lewbel R. (1997), «Quadratic Engel Curves and Consumer Demand », The Review of Economics and Statistics, vol. 79, novembre, pp. 527- 38.

Cogneau D., Razafindrakoto M. et Roubaud F. (1996), «Le secteur informel urbain et l'ajustement structurel au Cameroun », Revue d'Economie du Développement, n³, pp. 27-63.

Cremer H., Gahvari F. (1993), « Tax evasion and optimal commodity taxation », Journal of Public Economics, vol 50, pp $261-275$.

Deaton A. (1997), The analysis of household surveys : a microeconometric approach to development policy, Ed. The John Hopkins University Press, The World Bank, 497 p.

Deaton A. et Grimard F. (1993), «Demand analysis and tax reform in Pakistan», LSMS Working Paper n ${ }^{\circ}$, World Bank, 45 p.

Deaton A. et Muellbauer J. (1980), «An Almost Ideal Demand System», The American Economic Review, vol 70, n³, pp. 312- 26.

Fortin B., Marceau N., Savard L. (1997), « Taxation, wage controls and the informal sector », Journal of Public Economics, vol 66, pp 293 - 312.

Gautier J-F. (1999), «Les paradoxes de la TVA dans un pays en développement : l'illustration de l'industrie malgache », Tiers Monde, vol 40, n 157, pp. 187-211.

Goaïed M. (1991), «Estimation d'un système de demande des ménages à partir de données groupées des enquêtes budgétaires en Tunisie », Economie et Prévision, n97, pp. 11-19.

Kaplow L. (1990), «Optimal Taxation with costly enforcement and evasion », Journal of Public Economics, vol 43, pp $221-236$.

MADIO (1995a), Le secteur informel dans l'agglomération d'Antananarivo. Performances, Insertion, Perspectives. Enquête 1-2-3, phase 2, 50 p.

MADIO (1995b), La consommation et le rôle du secteur informel dans la satisfaction des ménages dans l'agglomération d'Antananarivo, Enquête 1-2-3, phase 3, 33 p.

Nichèle V. et Robin J-M. (1993), «Evaluation des effets redistributifs des réformes de la fiscalité indirecte française », Economie et Prévision, n 110-111, pp. 105-28. 
Ravelosoa J.R., Hagglabe S. et Rajemison H. (1999), «Estimation des élasticités de la demande à Madagascar à partir d'un modèle AIDS », Institut National de la Statistique de Madagascar, octobre, $52 \mathrm{p}$.

Ray R. (1997), «Issues in the design and reform of commodity taxes : analytical results and empirical evidence », Journal of Economic Surveys, vol 11, n4, pp. 353 - 88.

Roubaud F. (1994), «Consommation et condition de vie des ménages à Yaoundé », DIAL -DSCN, $\mathrm{n}^{\circ} 1994-04 \mathrm{E}$. 
ANNEXE

Annexe $n^{\circ} 1$ : Variation de la demande et des prix

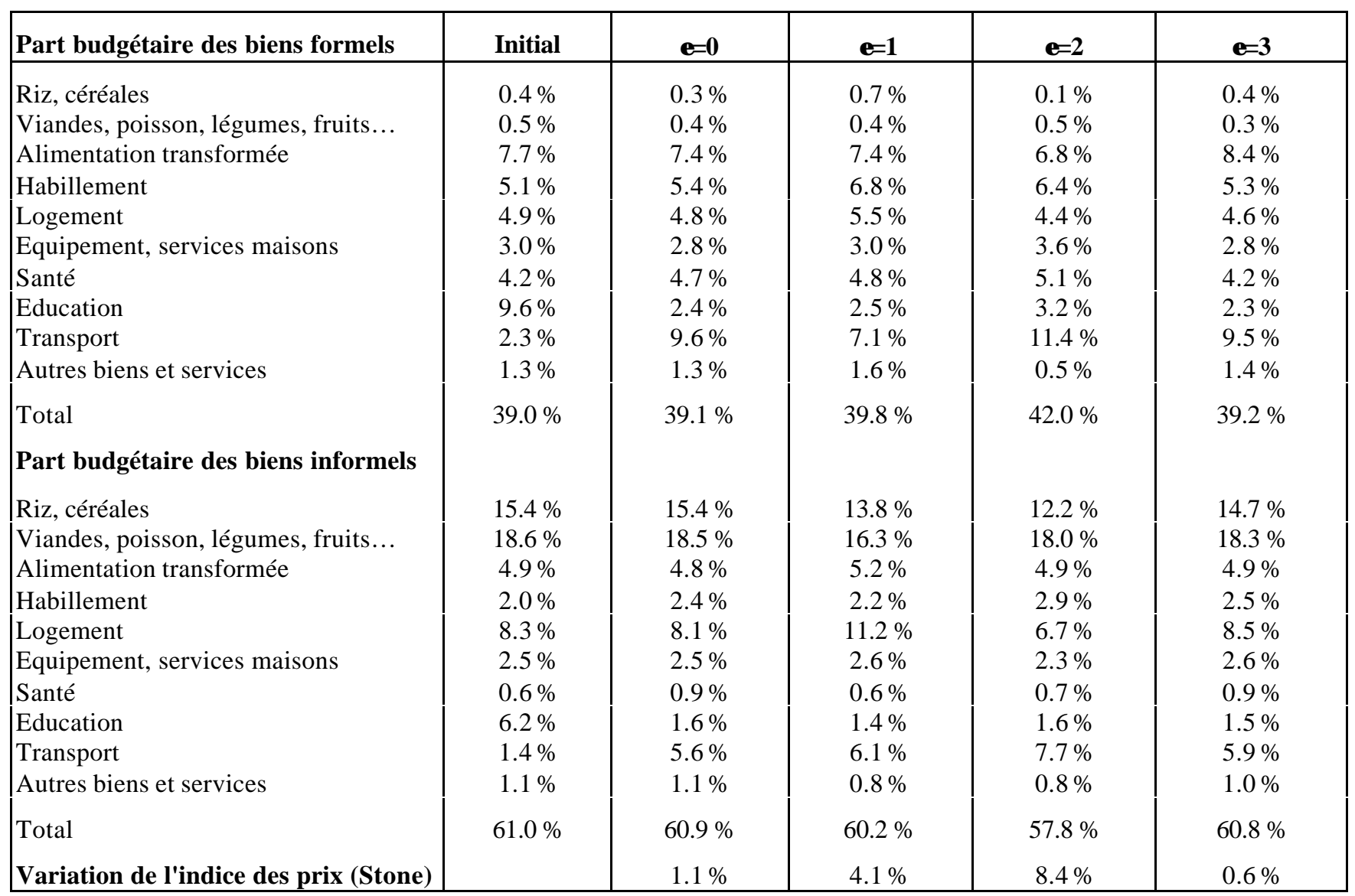

Annexe $n^{\circ} 2$ : Structure de la consommation et taux de pression par déciles de dépenses

\begin{tabular}{|c|c|c|c|c|}
\hline \multirow{2}{*}{$\begin{array}{c}\text { Déciles de } \\
\text { Dépenses }\end{array}$} & \multicolumn{3}{|c|}{ Taux de TVA effectif } & \multirow{2}{*}{$\begin{array}{c}\text { Coefficient de } \\
\text { Variation }\end{array}$} \\
\cline { 2 - 5 } & Moyen & Minimum & Maximum & $10.3 \%$ \\
2 & $2.0 \%$ & $0.4 \%$ & $6.2 \%$ & $8.7 \%$ \\
3 & $1.9 \%$ & $0.3 \%$ & $5.5 \%$ & $9.4 \%$ \\
5 & $2.2 \%$ & $0.4 \%$ & $5.4 \%$ & $7.3 \%$ \\
6 & $2.4 \%$ & $0.8 \%$ & $6.0 \%$ & $6.7 \%$ \\
7 & $2.2 \%$ & $0.4 \%$ & $5.0 \%$ & $8.1 \%$ \\
8 & $2.4 \%$ & $0.4 \%$ & $6.5 \%$ & $6.7 \%$ \\
9 & $2.6 \%$ & $0.6 \%$ & $4.7 \%$ & $7.2 \%$ \\
& $2.6 \%$ & $0.7 \%$ & $5.9 \%$ & $9.0 \%$ \\
\hline
\end{tabular}

Sources : Centre Fiscale des Entreprises, MADIO, calculs propres, estimés à partir des données fiscales de l'année 1998. 